\title{
OBSERVACIONES SOBRE LOS ENCINOS DE BAJA CALIFORNIA
}

\author{
Por Annetta Carter *
}

Desde hace mucho tiempo se ha creído que había solamente tres especies de encino (Quercus) en Baja California, las cuales fueron publicadas por Goldman en el año 1916 como especies endémicas. Son Q. Brandegei, $Q$. devia y $Q$. idonea. Además, se creía que no existían encinos en la península entre la región del Cabo, que era probablemente una isla durante la época del Plioceno, y la Sierra San Pedro Mártir en la parte norte. Pero en los años recientes ha aumentado nuestro conocimiento de los encinos. Desde el año 1947 me he ocupado del estudio de la flora de Baja California, especialmente la de la Sierra Giganta, y cada año he visitado la península para estudiarla. Los profesores C. H. Muller de la Universidad de California, Santa Bárbara, y J. M. Tucker de la misma Universidad, Davis, California, me hicieron el favor de revisar mi identificación de algunos de estos encinos y de identificar otros, y el Sr. R. E. K. Peters del Herbario de la Universidad de California, Berkeley, aumentó la información sobre $Q$. tuberculata ( $Q$. idonea) en la región al sur de La Paz.

QUERCUS BRANDEGEI Goldman, Contr. U. S. Nat. Herb. 16:321. 1916. (Subgénero Lepidobalanus.) "Encino negro". Esta especie es un árbol grande con hojas persistentes. Se encuentra a una altura de 150 hasta $500 \mathrm{~m}$. a lo largo de arroyos en las vertientes del Océano Pacífico y las del Golfo de Baja California en la región de la Sierra de la Victoria al sur de La Paz. Debajo de los árboles en las cercanías del Rancho San Juan de Acerraderra $(22.4 \mathrm{~km}$. al este de Todos Santos) se puede ver el suelo cubierto por retoños, como los ha descrito el Dr. Muller (The significance of vegetative reproduction in Quercus, Madroño 11:129-136. 1951). Las bellotas son de hasta unos $3-4 \mathrm{~cm}$. de largo con una punta aguda y de sabor sólo ligeramente amargo. Se venden en las tiendas de $\mathrm{La} \mathrm{Paz}$ como comestibles.

QUERCUS DEVIA Goldman, Contr. U. S. Nat. Herb. 16:322. 1916. (Subgénero Erythrobalanus.) "Encino negro". Abunda esta especie en la proximidad de La Laguna, Sierra de la Victoria, a una altura de 1,700 m.

* Department of Botany, University of California, Berkeley. 
En "La Aguja" de la misma Sierra lo encontré hasta una altura de 1,900 m. y en Arroyo Santa Rita al oeste de Santiago abunda con Q. tuberculata a una altura de $850 \mathrm{~m}$. hasta la cumbre $(1,800 \mathrm{~m}$.) cerca de La Chuparrosa donde recogió el tipo el Sr. Goldman. Donde los árboles crecen juntos son derechos y delgados, pero los árboles aislados son grandes y extendidos. Las hojas no son caducas pero caen a medida que envejecen.

QUERCUS TUBERCULATA Liebm. Overs. Dansk. Vid. Selsk. For. 1854. 181. 1854. Q. idonea Goldman, Contr. U. S. Nat. Herb. 16:321. 1916. (Subgénero Lepidobalanus.) "Encino roble". Aunque Goldman describió $Q$. idonea como uno de los encinos endémicos de la parte sur de Baja California, lo consideramos idéntico con el $Q$. tuberculata de Sonora y Chihuahua. Es el encino que se encuentra más frecuentemente en la parte sur de Baja California.Existe en mesas quebradas a una altura de 500 hasta $600 \mathrm{~m}$. y también en las montañas a una altura de 850 hasta $1,800 \mathrm{~m}$. Las hojas son caducas; aparecen en el verano después de las lluvias y suelen caer en la primavera hacia los meses de abril y mayo. El tamaño de las hojas es muy variable. Arboles que crecen en sitios altos y descubiertos tienen hojas pequeñas y coriáceas y de sólo 3 ó $5 \mathrm{~cm}$. de largo, mientras que los árboles de lugares abrigados o favorables tienen hojas delgadas y de hasta $12 \mathrm{~cm}$. de largo. De vez en cuando se encuentra un árbol con hojas muy grandes de hasta $16 \mathrm{~cm}$. de largo.

Ahora sabemos que existe este encino al norte de $\mathrm{La} \mathrm{Paz}$ en Baja California hasta el Cerro de La Giganta al oeste de Loreto (lat. 26॰8' N., long. $11^{\circ} 35^{\prime}$ W.). Aquí se encuentra desde una altura de $700 \mathrm{~m}$. cåsi hasta la cumbre del Cerro a una altura de 1,738 m. (Gentry 4147, 4290; Carter, Alexander \& Kellog 2009, 2011, 2031, Carter \& Ferris 3433). Es de interés notar que Dodonaea viscosa, Nolina Beldigi y Notholaena aurea, especies que crecen alrededor de Quercus tuberculata en La Laguna, también están aquí con él en la cumbre del Cerro de la Giganta. No se les ha encontrado en la zona situada entre los dos lugares.

QUERGUS RETICULATA Humb. y Bonpl. Pl. Aequin. 2:40. 1809. (Subgénero Lepidobalanus.) "Encino blanco". Encontramos estos encinos blancos en un cañón angosto y profundo al lado norte de La Laguna, Sierra de la Victoria (Carter, Alexander \& Kellog 2339, diciembre, 1947; $\boldsymbol{R}$. E. K. Peters 257, mayo, 1948). No se ha encontrado antes en Baja California, pero $Q$. reticulata es bastante conocido en la Sierra Madre Occidental de México. Las hojas son grandes (hasta $16 \mathrm{~cm}$. de largo) y muy rugosas.

QUERCUS OBLONGIFOLIA Torr. in Sitgreaves, Rep. Zuñi and Col. 173. 1853. (Subgénero Lepidobalanus.) "Encino". En la primavera del año 1953, un amigo mío, el Sr. Howard Gulik de Glendale, California, se interesó en visitar el sitio de la Misión Guadalupe al noroeste de Mulegé, y también en ver los "güeribos" (Populus Brandegeei) que crecen en un arroyo en las cercanías del Rancho San Sebastián. A lo largo del sendero entre Rancho San Sebastián y Guadalupe encontró unos cuantos encinos y me trajo algunas hojas. Parecían de Quercus oblongifolia. En noviembre del mismo año fuimos yo y una amiga mía, la Srta. Louise Kellogg, para ave- 

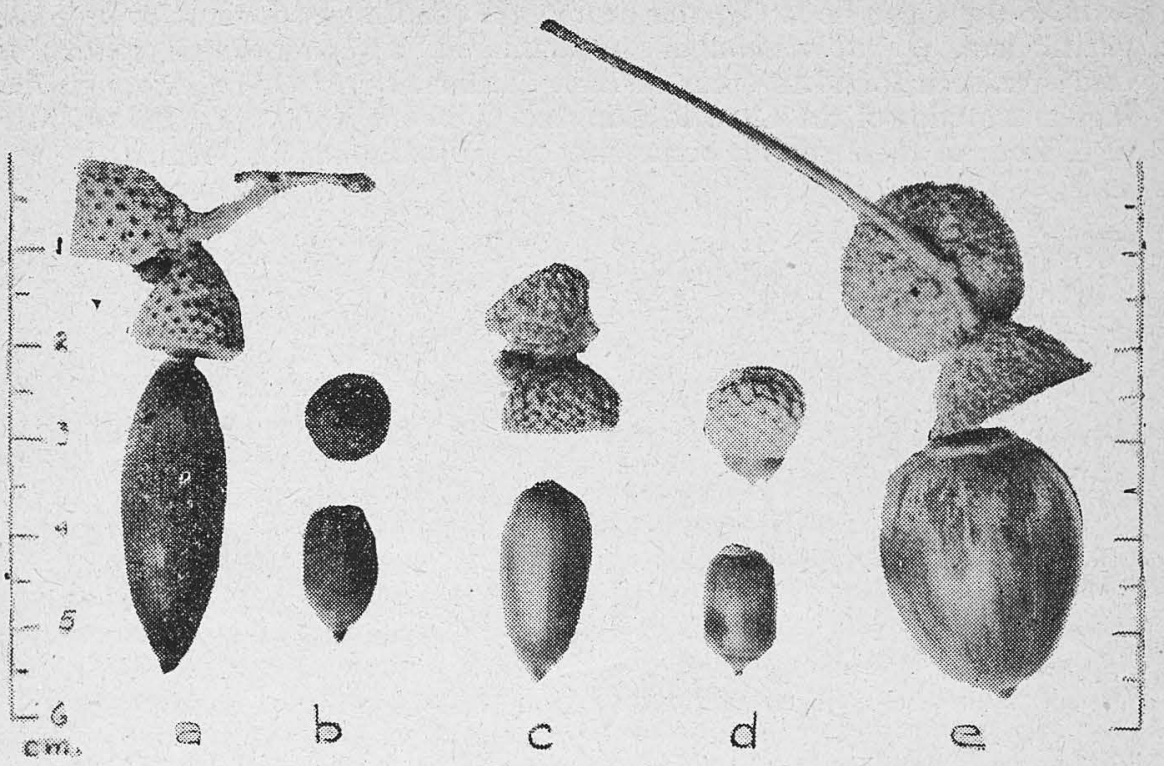

Fig. 1. Bellotas de los encinos de Baja California Sur: a, Quercus Brandegeei (Carter, Alexander \& Kellogg 2173; b, Q. devia (Brandegee, Oct. 2, 1899); $c$, Q. tuberculata (Carter, Alexander \& Kellogg 2178); d, Q. oblongifolia (Carter \& Kellogg 3271); e, Q. reticulata (Carter, Alexander \& Kellogg 2339). (Tamaño natural.)

riguar si eran $Q$. oblongifolia o no. A una altura de 1,100-1,200 m. había una docena o más de los árboles (Carter \& Kellogg 3271) que crecían en el nacimiento de un arroyo cerca de la cumbre de la cuesta (lat. 26 $57^{\prime} \mathrm{N}$., long. $112^{\circ} 25.5^{\prime}$ W.). Algunos de los árboles tenían bellotas. Eran verdaderamente $Q$. oblongifolia, especie que no se había encontrado antes en $\mathrm{Ba}$ ja California aunque existe con frecuencia al otro lado del Golfo en Sonora y Chihuahua, México, y en Arizona, E. U. del Norte. Es de interés notar que sobre las hojas había agallas de un insecto y que al sur de Nogales encontramos la misma clase de agallas sobre las hojas de $Q$. oblongifolia.

\section{S U M M A R Y}

Until recently the only oaks known to occur south of the San Pedro Mártir in Baja California, Mexico, were the three described in 1916 by Goldman: Quercus Brandegei, Q. devia and Q. idonea, all of which were believed to be restricted to the Cape Region of Baja California. Recent field work and studies have extended the known range of $Q$. idonea north 
ward to the Cerro de la Giganta near Loreto and have shown it to be conspecific with $Q$. tuberculata of the mainland. Two additional species of oak have been found to occur in Baja California Sur: Q. reticulata in the high mountains of the Cape Region and $Q$. oblongifolia northwest of $\mathrm{Mu}$ legé. Both of these species occur also on the mainland of Mexico. 